\title{
On operators close to isometries
}

\author{
by \\ Sameer Chavan (Pune)
}

\begin{abstract}
We introduce and discuss a class of operators, to be referred to as operators close to isometries. The Bergman-type operators, 2-hyperexpansions, expansive $p$ isometries, and certain alternating hyperexpansions are main examples of such operators. We establish a few decomposition theorems for operators close to isometries. Applications are given to the theory of $p$-isometries and of hyperexpansive operators.
\end{abstract}

1. Preliminaries. In this paper, we discuss the following fundamental problems from single-variable operator theory. If $S$ in $\mathcal{B}(\mathcal{H})$ is a completely non-unitary left-invertible operator, under what conditions does

- $S^{*}$ admit a complete set of eigenvectors?

- $S$ possess the wandering subspace property?

- $S$ admit a complete set of almost eigenvectors?

Recall that an operator $S$ in $\mathcal{B}(\mathcal{H})$ admits a complete set of eigenvectors if

$$
\mathcal{H}=\bigvee\{f \in \mathcal{H}:(S-\mu I) f=0 \text { for some } \mu \in \mathbb{C}\}
$$

and that $S$ possesses the wandering subspace property if

$$
\mathcal{H}=\bigvee_{n \geq 0} S^{n}\left(\mathcal{H} \cap(S \mathcal{H})^{\perp}\right) .
$$

A left-invertible operator $S$ in $\mathcal{B}(\mathcal{H})$ admits a complete set of almost eigenvectors if

$$
\mathcal{H}=\bigvee\left\{f \in \mathcal{H}:(S-\mu I) f \in \operatorname{null}\left(S^{*}\right) \text { for some } \mu \in \mathbb{C}\right\} .
$$

One of the main results of this paper asserts that if a completely nonunitary $S$ in $\mathcal{B}(\mathcal{H})$ is close to isometry, then $S^{*}$ admits a complete set of eigenvectors. Moreover, under some additional hypotheses, such an $S$ possesses the wandering subspace property as well as a complete set of almost

2000 Mathematics Subject Classification: Primary 47A16, 47B20.

Key words and phrases: hyponormal, hyperexpansive, hypercyclicity, $p$-isometric, wandering subspace. 
eigenvectors. We obtain these results as corollaries to certain decomposition theorems for operators close to isometries. Our work is partly motivated by the works of Clancey-Rogers ([7], [8]), Shimorin ([20]), and Yakubovich ([22]). Another motivation comes from the theory of $m$-isometries initiated by Agler-Stankus ([1], [2]), and of hyperexpansive operators initiated by Aleman ([3]) and Athavale-Sholapurkar ([5], [21]).

Unless stated otherwise, all the Hilbert spaces $\mathcal{H}$ occurring below are complex infinite-dimensional separable, and $\mathcal{B}(\mathcal{H})$ denotes the Banach algebra of bounded linear operators on $\mathcal{H}$. For any bounded linear operator $S$ on $\mathcal{H}$, we denote the spectrum, the point spectrum, the approximate point spectrum, the right spectrum, and the essential spectrum of $S$ by $\sigma(S), \sigma_{\mathrm{p}}(S)$, $\sigma_{\text {ap }}(S), \sigma_{\mathrm{r}}(S)$, and $\sigma_{\mathrm{e}}(T)$ respectively. The symbols null $(S)$ and $\operatorname{ran}(S)$ will stand for the null-space and the range-space of $S$ respectively. If $W$ is a subset of $\mathcal{H}$, then linspan $W$ will stand for the smallest linear manifold generated by $W$. By $\bigvee\{w: w \in W\}$, we mean the smallest closed linear manifold generated by $W$. For a subspace (that is, a closed linear manifold) $\mathcal{M}$ of $\mathcal{H}$, we use $P_{\mathcal{M}}$ to denote the orthogonal projection of $\mathcal{H}$ onto $\mathcal{M}$.

An operator $S$ in $\mathcal{B}(\mathcal{H})$ is said to be subnormal if there exist a Hilbert space $\mathcal{K}$ containing $\mathcal{H}$ and a normal operator $N$ in $\mathcal{B}(\mathcal{K})$ such that $N h=S h$ for every $h \in \mathcal{H}$. An operator $S$ in $\mathcal{B}(\mathcal{H})$ is hyponormal if the self-commutator $\left[S^{*}, S\right] \equiv S^{*} S-S S^{*}$ of $S$ is a positive operator. For the basic facts pertaining to subnormals and hyponormals, the reader is referred to [10]. Recall that a subnormal operator as well as the restriction of a hyponormal operator to an invariant subspace is hyponormal.

Let $m$ be a positive integer and let $S$ be in $\mathcal{B}(\mathcal{H})$. The operator $S$ is said to be completely hyperexpansive if

$$
B_{n}(S) \equiv \sum_{0 \leq p \leq n}(-1)^{p}\left(\begin{array}{l}
n \\
p
\end{array}\right) S^{* p} S^{p} \leq 0 \quad \text { for all integers } n \geq 1 .
$$

If $B_{n}(S) \leq 0$ for $1 \leq n \leq m$ then $S$ is said to be m-hyperexpansive. If $B_{m}(S)=0$ then $S$ is said to be m-isometric. $S$ is said to be expansive (respectively isometric) if it is 1-expansive (respectively 1-isometric). $S$ is said to be alternatingly hyperexpansive if

$$
C_{n}(S) \equiv \sum_{0 \leq p \leq n}(-1)^{p-n}\left(\begin{array}{l}
n \\
p
\end{array}\right) S^{* p} S^{p} \geq 0 \quad \text { for all integers } n \geq 1 .
$$

For the basic facts pertaining to $m$-isometries, $m$-hyperexpansions and alternating hyperexpansions, the reader is referred to [2], [5], [16], [20] and [21].

An operator $T$ in $\mathcal{B}(\mathcal{H})$ is said to be of Bergman type if

$$
\|T x+y\|^{2} \leq 2\left(\|x\|^{2}+\|T y\|^{2}\right) \quad(x, y \in \mathcal{H}) .
$$


Finally, if $S$ in $\mathcal{B}(\mathcal{H})$ is left-invertible then the operator $S^{\prime}$ given by $S^{\prime} \equiv$ $S\left(S^{*} S\right)^{-1}$ is said to be the operator Cauchy dual to $S$.

The paper is organized as follows. In the second section, we discuss several examples of almost isometric restriction classes, and record a few basic spectral properties of operators close to isometries (that is, the members of almost isometric restriction classes). In the third section, we establish our first decomposition theorem for operators close to isometries. As a consequence, we observe that a completely non-unitary operator close to isometry with $m$-dimensional cokernel belongs to the Cowen-Douglas class $\mathcal{B}_{m}\left(\mathbb{D}_{r}\right)$ for some positive real $r$. In the fourth section, we establish a Wold-type decomposition theorem for operators close to isometries. As a consequence, we obtain a general Beurling-type theorem of which the Beurling-type theorems for the Dirichlet shift and for the Bergman shift are special cases. We conclude the paper with some generalizations and examples.

2. Operators close to isometries. Let ISO denote the class of bounded linear isometries on Hilbert spaces. Note that the restriction of a member of ISO to a non-zero invariant subspace belongs to ISO. Also, the invertible members of ISO are precisely the unitaries. Moreover, the invariant subspaces $\mathcal{M}$ of isometries $S$ for which the restriction operator $\left.S\right|_{\mathcal{M}}$ turns out to be unitary are reducing. As we will see soon, many more classes other than ISO enjoy the same properties. We take these characteristic properties as part of the following definition.

Definition 2.1. A class $\mathcal{U}$ of left-invertible bounded linear operators on Hilbert spaces is said to be a restriction class if for every non-zero invariant subspace $\mathcal{M}$ of a bounded linear operator $S$ in $\mathcal{B}(\mathcal{H})$, the restriction operator $\left.S\right|_{\mathcal{M}}$ belongs to $\mathcal{U}$ whenever $S$ belongs to $\mathcal{U}$.

We are interested in those restriction classes $\mathcal{U}$ for which the following hold.

Property I. If $S$ belongs to $\mathcal{U}$, and $S$ is invertible, then $S$ is unitary.

Property II. If $S$ belongs to $\mathcal{U}$, and $\mathcal{M}$ is a non-zero invariant subspace of $S$ such that $\left.S\right|_{\mathcal{M}}$ is unitary, then $S$ reduces $\mathcal{M}$.

A restriction class $\mathcal{U}$ is said to be an almost isometric restriction class (for short, an AIR class) if it has Properties I and II. We will refer to the members of AIR classes as operators close to isometries.

Remark 2.2. Suppose $T$ in $\mathcal{B}(\mathcal{H})$ is close to isometry. Since a normal left-invertible operator is invertible, it follows from Property I that $T$ is completely non-normal (that is, $T$ has no non-trivial normal direct summand) if 
and only if $T$ is completely non-unitary (that is, $T$ has no non-trivial unitary direct summand).

ExAmple 2.3. Let $\mathbb{D}_{1}$ and $\partial \mathbb{D}_{1}$ denote the open unit disc $\{\mu \in \mathbb{C}:|\mu|<1\}$ and the unit circle, respectively. Consider the following classes.

(1) $\mathcal{U}_{1} \equiv\{T: T$ is of Bergman type $\}$.

(2) $\mathcal{U}_{2} \equiv\{T: T$ is 2-hyperexpansive $\}$.

(3) $\mathcal{U}_{3} \equiv\left\{T: T\right.$ is hyponormal such that $\left.\sigma_{\mathrm{ap}}(T) \subset \partial \mathbb{D}_{1}\right\}$.

(4) $\mathcal{U}_{4} \equiv\{T: T$ is an expansive $p$-isometry for some positive integer $p\}$.

(5) $\mathcal{U}_{5} \equiv\left\{T: T\right.$ is alternatingly hyperexpansive such that $\left.\sigma(T) \subset \overline{\mathbb{D}}_{1}\right\}$.

Then all the above are AIR classes.

Verification. Suppose $T \in \mathcal{U}_{1}$. It follows from Corollary 2.12 of [6] that $T$ is a hyponormal contraction. Moreover, since the operator Cauchy dual to $T$ is a 2-hyperexpansion ([20, proof of Theorem 3.6]), by Lemma 2.14 and Remark 2.4 of [6], one has $\sigma_{\text {ap }}(T) \subset \partial \mathbb{D}_{1}$. This shows that $\mathcal{U}_{1} \subset \mathcal{U}_{3}$. Since a subclass of an AIR class is again an AIR class, it suffices to check that $\mathcal{U}_{2}, \ldots, \mathcal{U}_{5}$ are AIR classes. It either follows from the definition or some well-known fact that $\mathcal{U}_{2}, \mathcal{U}_{3}$, and $\mathcal{U}_{4}$ are restriction classes. To see that $\mathcal{U}_{5}$ is a restriction class, let $T$ be alternatingly hyperexpansive such that $\sigma(T) \subset \overline{\mathbb{D}}_{1}$ and let $\mathcal{M}$ be any subspace invariant for $T$. Clearly, $\left.T\right|_{\mathcal{M}}$ is alternatingly hyperexpansive. Also, since $\sigma_{\text {ap }}\left(\left.T\right|_{\mathcal{M}}\right) \subset \sigma_{\text {ap }}(T)$, one has $\sigma_{\text {ap }}\left(\left.T\right|_{\mathcal{M}}\right) \subset \sigma_{\text {ap }}(T) \subset \overline{\mathbb{D}}_{1}$. Since the boundary of the spectrum of a bounded linear operator is contained in its approximate point spectrum, we must have $\sigma\left(\left.T\right|_{\mathcal{M}}\right) \subset \overline{\mathbb{D}}_{1}$. Hence $\left.T\right|_{\mathcal{M}}$ belongs to $\mathcal{U}_{5}$.

Next we check that the members of $\mathcal{U}_{2}, \ldots, \mathcal{U}_{5}$ have Property I. Suppose $T \in \mathcal{U}_{3}$. Since the boundary of the spectrum of a bounded linear operator is contained in its approximate point spectrum, invertibility of $T$ together with $\sigma_{\text {ap }}(T) \subset \partial \mathbb{D}_{1}$ forces $\sigma(T) \subset \partial \mathbb{D}_{1}$. Hence by the Putnam inequality ([10, Corollary 3.2 of Chapter IV]), an invertible $T$ in $\mathcal{U}_{3}$ must be normal. Hence such a $T$ is unitary and $\mathcal{U}_{3}$ has Property I. Also, it is noted in [21, Remark 3.4] that an invertible 2-hyperexpansive $T$ is unitary, so that $\mathcal{U}_{2}$ has Property I. Suppose $T$ is an invertible expansive $p$-isometry. It follows that $T^{-1}$ is a contractive $p$-isometry. We claim that $T^{-1}$ is isometric. We will derive this from the following general fact.

Lemma 2.4. If $S$ in $\mathcal{B}(\mathcal{H})$ is a contraction then for any $n \geq 1$, one has

$$
B_{n+1}(S) \leq 0 \Rightarrow B_{n}(S) \leq 0,
$$

where $B_{m}(S)=\sum_{0 \leq p \leq m}(-1)^{p}\left(\begin{array}{c}m \\ p\end{array}\right) S^{* p} S^{p}(m \geq 1)$.

Proof. Suppose that $S$ in $\mathcal{B}(\mathcal{H})$ is a contraction, and $B_{n+1}(S) \leq 0$ for some $n \geq 1$. Since $S$ is a contraction, there exists a positive operator $A$ in 
$\mathcal{B}(\mathcal{H})$ such that

$$
S^{* n} S^{n} \rightarrow A(\mathrm{SOT}) \quad \text { as } n \rightarrow \infty
$$

(see [17]). Also, since $B_{n+1}(S)=B_{n}(S)-S^{*} B_{n}(S) S$, one has $B_{n}(S) \leq$ $S^{*} B_{n}(S) S$. An induction argument shows that $B_{n}(S) \leq S^{* m} B_{n}(S) S^{m}$ $(m \geq 1)$. Thus it suffices to check that $S^{* m} B_{n}(S) S^{m} \rightarrow 0$ (SOT) as $m \rightarrow \infty$. Note that

$$
\begin{aligned}
B_{n}(S) & =B_{n-1}(S)-S^{*} B_{n-1}(S) S \\
& =\sum_{0 \leq p \leq n-1}(-1)^{p}\left(\begin{array}{c}
n-1 \\
p
\end{array}\right)\left(S^{* p} S^{p}-S^{* p+1} S^{p+1}\right),
\end{aligned}
$$

so that

$$
S^{* m} B_{n}(S) S^{m}=\sum_{0 \leq p \leq n-1}(-1)^{p}\left(\begin{array}{c}
n-1 \\
p
\end{array}\right)\left(S^{* p+m} S^{p+m}-S^{* p+m+1} S^{p+m+1}\right)
$$

for every integer $m \geq 1$. By (2.1), a passage $m \rightarrow \infty$ leads to

$$
S^{* m} B_{n}(S) S^{m} \rightarrow \sum_{0 \leq p \leq n-1}(-1)^{p}\left(\begin{array}{c}
n-1 \\
p
\end{array}\right)(A-A)(\mathrm{SOT})=0
$$

Now we prove the claim. Since $T^{-1}$ is a $p$-isometry, we have $B_{p}\left(T^{-1}\right)=0$. In particular, $B_{p}\left(T^{-1}\right) \leq 0$. Also, since $T^{-1}$ is a contraction, by repeated applications of Lemma 2.4, one has $B_{1}\left(T^{-1}\right) \leq 0$, that is, $T^{-1}$ is expansive. Hence $T^{-1}$ is isometric, and therefore $T$ is unitary.

Next, assume that $T$ is an invertible alternating hyperexpansion and that $\sigma(T) \subset \overline{\mathbb{D}}_{1}$. Since an invertible alternating hyperexpansion is subnormal ([21, Remark 4.2]), and since norm and spectral radius coincide for subnormals ([10]), the invertibility of $T$ together with $\sigma(T) \subset \overline{\mathbb{D}}_{1}$ forces $T$ to be a contraction. Hence $T$ is an invertible isometry, that is, a unitary.

To see that all the classes under consideration have Property II, we need the following simple lemma (cf. [20, Lemma 3.3], [6, proof of Proposition 2.17]).

Lemma 2.5. If $S$ in $\mathcal{B}(\mathcal{H})$ is either contractive or expansive, and if $\mathcal{M}$ is a non-zero invariant subspace of $S$ such that $\left.S\right|_{\mathcal{M}}$ is unitary, then $S$ reduces $\mathcal{M}$.

Proof. Note that $P_{\mathcal{M}} S^{*} S h=h$ for any $h \in \mathcal{M}$, where $P_{\mathcal{M}}$ is the orthogonal projection from $\mathcal{H}$ onto $\mathcal{M}$. Therefore for any $h \in \mathcal{M},\left\langle h-S^{*} S h, h\right\rangle_{\mathcal{H}}=$ $\left\langle h-P_{\mathcal{M}} S^{*} S h, h\right\rangle_{\mathcal{M}}=0$. If $S$ is contractive then $\left\|\left(I-S^{*} S\right)^{1 / 2} h\right\|_{\mathcal{H}}=0$ for any $h \in \mathcal{M}$. If $S$ is expansive then $\left\|\left(S^{*} S-I\right)^{1 / 2} h\right\|_{\mathcal{H}}=0$ for any $h \in \mathcal{M}$. In any case, $S^{*} S h=h$ for any $h \in \mathcal{M}$. This observation together with $S \mathcal{M}=\mathcal{M}$ leads to $S^{*} \mathcal{M}=\mathcal{M}$. Thus $S$ reduces $\mathcal{M}$. 
Since the spectral radius of a hyponormal operator is equal to its norm ([10]), it follows that the members of $\mathcal{U}_{3}$ are contractive. It is now clear that the members of all classes under consideration are either contractions or expansions. Hence by the preceding lemma, Property II holds for all such classes. This completes the verification of Example 2.3.

We conclude the section with a brief discussion of the spectral behavior of operators close to isometries.

Lemma 2.6. Let $T \in \mathcal{B}(\mathcal{H})$ be completely non-unitary and close to isometry. For $r>0$ let $\mathbb{D}_{r}$ denote $\{z \in \mathbb{C}|| z \mid<r\}$. Then there exists $r_{0}>0$ such that

(a) $\mathbb{D}_{r_{0}} \subset \sigma_{p}\left(T^{*}\right)$.

(b) $\operatorname{ran}\left(T^{*}-\mu I\right)=\mathcal{H}\left(\mu \in \mathbb{D}_{r_{0}}\right)$.

(c) $\operatorname{nullity}(T-\mu I)=\operatorname{nullity}(T)\left(\mu \in \mathbb{D}_{r_{0}}\right)$, where nullity $(S)$ stands for the dimension of the null-space of the bounded linear operator $S$.

Proof. Since $T$ is completely non-unitary, $0 \in \sigma(T)$ by Property I. Since $T$ is left-invertible, there exists $r_{0}>0$ such that $\|T x\| \geq r_{0}\|x\|$ for every $x \in \mathcal{H}$. Hence by a standard argument, $\|(T-\mu I) x\| \geq\left(r_{0}-|\mu|\right)\|x\|$ for all $\mu \in \mathbb{D}_{r_{0}}$ and $x \in \mathcal{H}$. This shows that $\mathbb{D}_{r_{0}} \cap \sigma_{\text {ap }}(T)=\emptyset$. Now the desired assertions can be concluded from the standard spectral and Fredholm theory ([17], [10]).

REMARK 2.7. In the case where $\mathcal{U}$ is one of $\mathcal{U}_{1}, \ldots, \mathcal{U}_{5}$ (see Example 2.3), for a completely non-unitary $T$ in $\mathcal{U}$, the positive real $r_{0}$ in the conclusions (a), (b), (c) of Lemma 2.6 can be chosen to be 1. Indeed, in this case $\sigma(T)=$ $\overline{\mathbb{D}}_{1}$ (see [2, Lemma 1.21], [21, Remark 2.4], [6, Lemma 2.14], [17]).

The author does not know a single example of a completely non-unitary operator close to isometry with spectrum different from the closed unit disc $\overline{\mathbb{D}}_{1}$. In view of this and Remark 2.7, the following question is interesting.

Question. If $T$ is close to isometry, does the following spectral dichotomy hold: if $T$ is invertible then $\sigma(T) \subset \partial \mathbb{D}_{1}$, and if not then $\sigma(T)=\overline{\mathbb{D}}_{1}$ ?

3. Completeness of eigenvectors. In this section, we establish our first decomposition theorem (cf. [7]) for operators close to isometries. As a consequence, we observe that the $m$-multicyclic completely non-unitary operators which are close to isometries belong to the Cowen-Douglas class $B_{m}\left(\mathbb{D}_{r}\right)$ for some $r>0$. Also, we observe that for a completely non-unitary operator $T$ which is close to isometry, the operator $\alpha T^{*}$ turns out to be hypercyclic for any complex $\alpha$ of modulus greater than 1 provided $\sigma\left(T^{\prime}\right) \subset$ $\overline{\mathbb{D}}_{1}$. We begin with a lemma, which is definitely known (see, for example, [7]). 
Lemma 3.1. Let $T$ in $\mathcal{B}(\mathcal{H})$ be such that $\|T x\| \geq r_{0}\|x\|(x \in \mathcal{H})$ for some $r_{0}>0$. Define $P(\cdot): \mathbb{D}_{r_{0}} \rightarrow \mathcal{B}(\mathcal{H})$ by $P(\mu)=P_{\text {null }\left(T^{*}-\mu I\right)}\left(\mu \in \mathbb{D}_{r_{0}}\right)$. Then $P(\cdot)$ is continuous at 0 in the uniform sense, that is, $\|P(\mu)-P(0)\| \rightarrow 0$ as $\mu \rightarrow 0$.

Proof. Consider the operator-valued function $R: \mathbb{D}_{r_{0}} \rightarrow \mathcal{B}(\mathcal{H})$ given by

$$
R(\mu)=(T-\bar{\mu} I)^{\prime *} \quad\left(\mu \in \mathbb{D}_{r_{0}}\right),
$$

where $(T-\bar{\mu} I)^{\prime}$ denotes the operator Cauchy dual to the left-invertible operator $T-\bar{\mu} I\left(\mu \in \mathbb{D}_{r_{0}}\right)$. Using the fact that the map $S \mapsto S^{-1}$ from the set $\mathcal{G}(\mathcal{L})$ of invertible operators on a Hilbert space $\mathcal{L}$ into $\mathcal{B}(\mathcal{L})$ is continuous in the norm topology $([9])$, it is easy to see that $R(\cdot)$ is continuous at 0 in the uniform sense. As $(T-\bar{\mu} I) R(\mu)=I-P_{\operatorname{null}\left(T^{*}-\mu I\right)}$ for every $\mu \in \mathbb{D}_{r_{0}}$, the assertion follows.

Theorem 3.2. Let $T$ be in $\mathcal{B}(\mathcal{H})$, and let $r>0$. If $T$ is close to isometry, then

$$
T=U \oplus C \quad \text { on } \mathcal{H}=\mathcal{H}_{\mathrm{u}} \oplus \mathcal{H}_{\mathrm{c}},
$$

where $U$ is unitary on $\mathcal{H}_{\mathrm{u}}, C$ is completely non-unitary on $\mathcal{H}_{\mathrm{c}}$, and $\mathcal{H}_{\mathrm{c}}$ is equal to $\bigvee\left\{\operatorname{null}\left(C^{*}-\mu I\right): \mu \in \mathbb{D}_{r}\right\}$.

Proof. Let $\mathcal{U}$ be an AIR class, and let $T$ be in $\mathcal{U} \cap \mathcal{B}(\mathcal{H})$. Let $r>0$ be given. Write

$$
T=U \oplus C \quad \text { on } \mathcal{H}=\mathcal{H}_{\mathrm{u}} \oplus \mathcal{H}_{\mathrm{c}},
$$

where $U$ is a normal operator on $\mathcal{H}_{\mathrm{u}}$ and $C$ is completely non-normal on $\mathcal{H}_{\mathrm{c}}$ ([18, Corollary 1.3]). Note that $U, C \in \mathcal{U}$. Hence by Remark 2.2, $C$ is completely non-unitary. Thus it suffices to show that $\mathcal{H}_{\mathrm{c}}=\bigvee\left\{\operatorname{null}\left(C^{*}-\mu I\right)\right.$ : $\left.\mu \in \mathbb{D}_{r}\right\}$. We adapt the argument of $([8$, Theorem 2]) to the present situation. Set $\mathcal{H}_{1} \equiv \bigvee\left\{\operatorname{null}\left(C^{*}-\mu I\right): \mu \in \mathbb{D}_{r}\right\}$. Thus

$$
C=\left(\begin{array}{cc}
C_{2} & 0 \\
X & C_{1}
\end{array}\right) \quad \text { on } \mathcal{H}_{\mathrm{c}}=\mathcal{H}_{1} \oplus \mathcal{H}_{1}^{\perp}
$$

Clearly, $C_{1}$ belongs to $\mathcal{U}$. In particular, $\operatorname{null}\left(C_{1}\right)=\{0\}$ and $\overline{\operatorname{ran}\left(C_{1}\right)}=$ $\operatorname{ran}\left(C_{1}\right)$. Next note that linspan $\left\{\operatorname{null}\left(C^{*}-\mu I\right): \mu \in \mathbb{D}_{r}, \mu \neq 0\right\} \subset \operatorname{ran}\left(C_{2}^{*}\right)$. Hence using the continuity of $P(\cdot)$ at 0 (Lemma 3.1), it can be easily seen that $\operatorname{null}\left(C^{*}\right) \subset \overline{\operatorname{ran}\left(C_{2}^{*}\right)}$. Thus $\overline{\operatorname{ran}\left(C_{2}^{*}\right)}=\mathcal{H}_{1}$ and so $\operatorname{null}\left(C_{2}\right)=\{0\}$.

We claim that $C_{1}$ is onto. Since $\operatorname{ran}\left(C_{1}\right)$ is closed, it suffices to check that $\operatorname{null}\left(C_{1}^{*}\right)=\{0\}$. To see the latter, we may check that $g$ is zero whenever $g \in \mathcal{H}_{\mathrm{c}}, C g \in \mathcal{H}_{1}^{\perp}$ and $C_{1}^{*} C g=0$. This follows by a routine calculation using $\operatorname{null}\left(C_{1}\right)=\{0\}$ and null $\left(C_{2}\right)=\{0\}$. Therefore $C_{1}^{*}$ is injective. It follows that $C_{1}$ is an invertible operator in $\mathcal{U}$. By Property I, $C_{1}$ is unitary. Also, since $C_{1}=\left.C\right|_{\mathcal{H}_{1}^{\perp}}$, by Property II, $C$ reduces $\mathcal{H}_{1}^{\perp}$. Since $C$ is completely non-unitary, we must have $\mathcal{H}_{\mathrm{c}}=\mathcal{H}_{1}$, and the proof is over. 
REMARK 3.3. In the case where $\mathcal{U}=\mathcal{U}_{3}$ (see Example 2.3), and $T$ in $\mathcal{U}$ is completely non-unitary, Theorem 3.2 recovers a special case of Theorem 2 of [8], which was proved under the hypotheses that $T$ is pure hyponormal and $\operatorname{Area}\left(\sigma_{\mathrm{ap}}(T)\right)=0$. On the other hand, our method applies to a number of classes including that of 2-hyperexpansions, expansive $p$-isometries, and certain alternating hyperexpansions.

We discuss below several applications of Theorem 3.2 to the theory of $p$ isometries and of hyperexpansive operators. We need some definitions. Let $m$ be a positive integer. An operator $S$ in $\mathcal{B}(\mathcal{H})$ is said to be finitely multicyclic if there are a finite number of vectors $h_{1}, \ldots, h_{m}$ in $\mathcal{H}$ such that

$$
\mathcal{H}=\bigvee\left\{S^{k} h_{1}, \ldots, S^{k} h_{m}: k \geq 0\right\} .
$$

$S$ is said to be $m$-multicyclic if $(3.2)$ holds for some set $\left\{h_{1}, \ldots, h_{m}\right\}$ of $m$ vectors in $\mathcal{H}$ but for no set of $m-1$ vectors. $S$ is cyclic if it is 1-cyclic. In that case, there exists a vector $e_{0}$ in $\mathcal{H}$ such that $\mathcal{H}=\bigvee\left\{S^{k} e_{0}: k \geq 0\right\}$. The vector $e_{0}$ is referred to as a cyclic vector for $S$.

Corollary 3.4. Let $T \in \mathcal{B}(\mathcal{H})$ be completely non-unitary and close to isometry. Then:

(a) For any positive real $r$, one has $\mathcal{H}=\bigvee\left\{\operatorname{null}\left(T^{*}-\mu I\right): \mu \in \mathbb{D}_{r}\right\}$.

(b) $T^{*}$ has a dense set of cyclic vectors.

Proof. (a) follows from the previous theorem. The other part follows from Theorem 3 of [8] provided we show that $\bigvee\left\{\operatorname{null}\left(T^{*}-\mu I\right): \mu \notin \sigma_{r}\left(T^{*}\right)\right\}=\mathcal{H}$. Since one can choose $r_{0}>0$ so that $\mathbb{D}_{r_{0}} \subset \mathbb{C} \backslash \sigma_{r}\left(T^{*}\right)$, the proof is over.

REMARK 3.5. Let $T \in \mathcal{B}(\mathcal{H})$ be completely non-unitary and close to isometry. Let $e_{0}$ in $\mathcal{H}$ be a cyclic vector for $T^{*}$ such that $\left\|e_{0}\right\|=1$ (Corollary 3.4). Let $\left\{e_{n}\right\}_{n \geq 0}$ be the orthonormal basis obtained from the GramSchmidt orthonormalization process applied to the linearly independent set $\left\{T^{* n} e_{0}: n \geq 0\right\}$. (In case $\left\{T^{* n} e_{0}: n \geq 0\right\}$ is linearly dependent, $\mathcal{H}$ is finitedimensional, and consequently $T$ is invertible. By Property I, $T$ is unitary. Since $T$ is completely non-unitary, we arrive at a contradiction.) Then it is easy to see that $T$ has an "almost" upper triangular matrix representation with respect to $\left\{e_{n}\right\}_{n \geq 0}$, in the sense that the only non-zero entries above the main diagonal are possibly on the super diagonal. This idea of "almost" upper triangularization is due to Halmos ([12, Corollary 3.12]).

An operator $S$ in $\mathcal{B}(\mathcal{H})$ is said to be hypercyclic or $S$-universal if its orbit $\left\{S^{n} h: n \geq 0\right\}$ is dense in $\mathcal{H}$ for some vector $h \in \mathcal{H}$. If $U$ is a unilateral shift, then $\alpha U^{*}$ is hypercyclic for every complex number $\alpha$ of modulus greater than 1 . Surprisingly, every completely non-unitary member of $\mathcal{U}_{i}$ enjoys the same property $(i=1,2,3)$. This can be deduced from Corollary 3.6(b) and Example 3.10 below. Recall that $S$ in $\mathcal{B}(\mathcal{H})$ is strongly stable if $\left\|S^{n} h\right\| \rightarrow 0$ as 
$n \rightarrow \infty$ for every $h \in \mathcal{H}$. The following corollary shows in particular that if $S$ is a completely non-unitary hyponormal operator such that $\sigma_{\mathrm{ap}}(S) \subset \partial \mathbb{D}_{1}$, then $S^{*}$ is strongly stable. This recovers a special case of Theorem 5.3 of [17].

Corollary 3.6. Let $T$ in $\mathcal{B}(\mathcal{H})$ be completely non-unitary and close to isometry. Let $T^{\prime}$ be the operator Cauchy dual to $T$. Then the following are true.

(a) There exists a linear manifold $\mathcal{M}$ invariant for $T^{*}$ and dense in $\mathcal{H}$ such that $\left.T^{*}\right|_{\mathcal{M}}$ is strongly stable. If in addition $T$ is power bounded (that is, $\sup _{n \geq 0}\left\|T^{n}\right\|<\infty$ ), then $T^{*}$ is strongly stable.

(b) If $\sigma\left(T^{\prime}\right) \subset \overline{\mathbb{D}}_{1}$ then $\alpha T^{*}$ is hypercyclic for any complex $\alpha$ of modulus greater than 1.

Proof. By Corollary 3.4, one has $\mathcal{H}=\bigvee\left\{\operatorname{null}\left(T^{*}-\mu I\right): \mu \in \mathbb{D}_{r}\right\}$ for every $r \in(0,1)$. Hence the linear manifold

$$
\mathcal{M}_{r} \equiv \operatorname{linspan}\left\{h \in \mathcal{H}: h \in \operatorname{null}\left(T^{*}-\mu I\right), \mu \in \mathbb{D}_{r}\right\}
$$

invariant for $T^{*}$ is dense in $\mathcal{H}$. Moreover, for every $h \in \operatorname{null}\left(T^{*}-\mu I\right)$, one has $\left\|T^{* n} h\right\|=|\mu|^{n}\|h\|\left(\mu \in \mathbb{D}_{r}\right)$. It is now clear that $\left\|T^{* n} h\right\| \rightarrow 0$ as $n \rightarrow \infty$, and hence $\left.T^{*}\right|_{\mathcal{M}}$ is strongly stable. If in addition $\sup _{n \geq 0}\left\|T^{n}\right\|<\infty$, then using the stability of $\left.T^{*}\right|_{\mathcal{M}_{r}}$ and the density of $\mathcal{M}_{r}$, it can be easily seen that $T^{*}$ is strongly stable. Hence (a) is proved.

To establish (b), we use the hypercyclicity criterion ([13, Theorem 2.2]). Assume that $\sigma\left(T^{\prime}\right) \subset \overline{\mathbb{D}}_{1}$. In view of the proof of (a), it suffices to check the following: For every $\alpha \in \mathbb{C}$ such that $|\alpha|>1$, there exists $r>0$ such that

$$
\left\|\alpha^{n} T^{* n} h\right\| \rightarrow 0, \quad\left\|\alpha^{-n} T^{\prime n} h\right\| \rightarrow 0 \quad \text { as } n \rightarrow \infty
$$

for every $h \in \mathcal{M}_{r}$. Let $\alpha \in \mathbb{C}$ be such that $|\alpha|>1$ and let $\varepsilon>0$ be such that $1+\varepsilon<|\alpha|$. Since $\lim _{n \rightarrow \infty}\left\|T^{\prime n}\right\|^{1 / n} \leq 1$, one can choose a positive integer $n_{0}$ such that $\left\|T^{\prime n} h\right\| \leq\|h\|(1+\varepsilon)^{n}$ for every $n \geq n_{0}$. Then it is clear that $\left\|\alpha^{-n} T^{\prime n} h\right\| \rightarrow 0$ as $n \rightarrow \infty(h \in \mathcal{H})$. Let $r_{0} \in\left(0,|\alpha|^{-1}\right)$. It follows that $\left\|\alpha^{n} T^{* n} h\right\| \rightarrow 0$ as $n \rightarrow \infty$ for every $h \in \mathcal{M}_{r_{0}}$.

For a connected open subset $\Omega$ of the complex plane $\mathbb{C}$ and a positive integer $m$, let $\mathcal{B}_{m}(\Omega)$ denote the class of operators $S$ in $\mathcal{B}(\mathcal{H})$ for which

$$
\begin{aligned}
\Omega & \subset \sigma(S), \quad \operatorname{ran}(S-\mu I)=\mathcal{H} & & (\mu \in \Omega), \\
\bigvee_{\mu \in \Omega} \operatorname{null}(S-\mu I) & =\mathcal{H}, \quad \operatorname{nullity}(S-\mu I)=m & & (\mu \in \Omega) .
\end{aligned}
$$

The classes $\mathcal{B}_{m}(\Omega)$ were introduced by Cowen and Douglas in [11].

Corollary 3.7. Let $T$ in $\mathcal{B}(\mathcal{H})$ be close to isometry. If $T$ is m-multicyclic and completely non-unitary, then there exists a positive real $r$ such that $T^{*}$ belongs to the Cowen-Douglas class $\mathcal{B}_{m}\left(\mathbb{D}_{r}\right)$. 
Proof. Since $T$ is $m$-multicyclic, nullity $\left(T^{*}\right)=m$ ([14]). Moreover, by Corollary 3.4, one has $\mathcal{H}=\bigvee\left\{\operatorname{null}\left(T^{*}-\mu I\right): \mu \in \mathbb{D}_{r}\right\}$ for all $r>0$. Now one may appeal to Lemma 2.6.

REMARK 3.8. It is clear from Remark 2.7 and the preceding corollary that if $T$ in $\mathcal{B}(\mathcal{H})$ belongs to one of the restriction classes $\mathcal{U}_{1}, \ldots, \mathcal{U}_{5}$, and if it is $m$-multicyclic and completely non-unitary, then $T^{*} \in \mathcal{B}_{m}\left(\mathbb{D}_{1}\right)$.

We conclude the section with one more application of Theorem 3.2. Recall that a left-invertible operator $S$ in $\mathcal{B}(\mathcal{H})$ admits a complete set of almost eigenvectors if

$$
\mathcal{H}=\bigvee\left\{f \in \mathcal{H}:(S-\mu I) f \in \operatorname{null}\left(S^{*}\right) \text { for some } \mu \in \mathbb{C}\right\} .
$$

Completeness of almost eigenvectors is important in the construction of bundle shift models of left-invertible operators (see [22]).

Definition 3.9. Let $\mathcal{U}, \mathcal{V}$ be restriction classes. Then the Cauchy map $\mathcal{C}$ from $\mathcal{U}$ into $\mathcal{V}$ is given by $\mathcal{C}(S)=S^{\prime}(S \in \mathcal{U})$, where $S^{\prime} \equiv S\left(S^{*} S\right)^{-1}$ is the operator Cauchy dual to $S$.

Example 3.10. Consider the AIR classes $\mathcal{U}_{1}, \mathcal{U}_{2}, \mathcal{U}_{3}$ of Example 2.3. Then the map $\mathcal{C}_{i, i+1}: \mathcal{U}_{i} \rightarrow \mathcal{U}_{i+1}$ given by $\mathcal{C}_{i, i+1}(T)=T^{\prime}\left(T \in \mathcal{U}_{i}\right)$ is a Cauchy map $(i=1,2)$.

Verification. It is noted in [20, proof of Theorem 3.6] that the operator Cauchy dual to an operator $T \in \mathcal{U}_{1}$ is 2-hyperexpansive. Hence $\mathcal{C}_{1,2}$ is a Cauchy map. The fact that $\mathcal{C}_{2,3}$ is a Cauchy map follows from Theorem 2.9 and Lemma 2.14 of [6].

Corollary 3.11. Let $\mathcal{U}, \mathcal{V}$ be AIR classes, and let $\mathcal{C}: \mathcal{U} \rightarrow \mathcal{V}$ be the Cauchy map. Let $T$ in $\mathcal{U} \cap \mathcal{B}(\mathcal{H})$ be completely non-unitary. Then the set of almost eigenvectors of $T$ is complete.

Proof. Let $T^{\prime}$ be the operator Cauchy dual to $T$, and let $f_{\mu} \in \operatorname{null}\left(T^{*}-\mu I\right)$ for a non-zero $\mu$ in $\mathbb{C}$. Since $T^{*} f_{\mu}=\mu f_{\mu}$, and $T T^{\prime *}=P_{\operatorname{ran}(T)}$, one has $T f_{\mu}=\mu^{-1} P_{\operatorname{ran}(T)} f_{\mu}$. Hence

$$
\left(\mu^{-1} I-T\right) f_{\mu}=\mu^{-1} P_{\operatorname{null}\left(T^{*}\right)} f_{\mu} .
$$

Thus

$$
\left(\mu^{-1} I-T\right) f_{\mu} \in \operatorname{null}\left(T^{*}\right) \quad \text { whenever } \quad\left(f_{\mu} \in \operatorname{null}\left(T^{*}-\mu I\right), \mu \neq 0\right) .
$$

Moreover, if for some $f \in \mathcal{H}$ and $\mu \neq 0$, the vector $\left(\mu^{-1} I-T\right) f$ belongs to $\operatorname{null}\left(T^{*}\right)$, then using the fact that $\operatorname{null}\left(T^{*}\right)=\operatorname{null}\left(T^{*}\right)$, one has $\mu^{-1} T^{\prime *} f-f=0$. Hence $f \in \operatorname{null}\left(T^{\prime *}-\mu I\right)$. Thus $f_{\mu} \in \operatorname{null}\left(T^{\prime *}-\mu I\right)$ if and only if $f_{\mu}$ is an almost eigenvector of $T$ corresponding to the almost 
eigenvalue $\mu^{-1}$. Hence the set

$$
\mathcal{G} \equiv \operatorname{linspan}\left\{f_{\mu} \in \operatorname{null}\left(T^{*}-\mu I\right): \mu \in \mathbb{C} \backslash\{0\}\right\}
$$

consists of precisely the almost eigenvectors of $T$ corresponding to non-zero almost eigenvalues. We claim that $\mathcal{G}$ is dense in $\mathcal{H}$.

Note that the Cauchy dual operator $T^{\prime}$ is completely non-unitary since $T$ is. Also, since $\mathcal{C}$ is a Cauchy map, $T^{\prime}$ belongs to the almost isometric restriction class $\mathcal{V}$. Hence by Corollary 3.4, the set of eigenvectors of $T^{\prime *}$ is complete. We conclude the proof by showing that a vector $h \in \mathcal{H}$ is zero whenever $\langle h, f\rangle=0$ for every $f \in \mathcal{G}$. To see that, let $h \in \mathcal{H}$ be such that $\langle h, f\rangle=0$ for every $f \in \mathcal{G}$. Thus for a non-zero $\mu,\left\langle h, P_{\text {null }\left(T^{\prime *}-\mu I\right)} f\right\rangle=0$ for every $f \in \mathcal{G}$. Since the projection-valued map $P$ given by

$$
P(\mu)=P_{\text {null }\left(T^{\prime *}-\mu I\right)} \quad\left(\mu \in \sigma\left(T^{* *}\right) \backslash \sigma_{r}\left(T^{\prime *}\right)\right)
$$

is continuous at 0 (Lemma 3.1), for any $f$ in null $\left(T^{*}\right)$, one has

$$
0=\left\langle h, P_{\operatorname{null}\left(T^{*}-\mu I\right)} f\right\rangle \rightarrow\langle h, f\rangle \quad \text { as } \mu \rightarrow 0,
$$

hence $\langle h, f\rangle=0$ for every eigenvector of $T^{*}$. Since the set of eigenvectors of $T^{*}$ is complete, $h=0$, and the desired conclusion follows.

4. The wandering subspace property. In this section, we establish a Wold-type decomposition theorem for operators close to isometries under some additional assumptions. The proof is based on the technique developed by Shimorin in his work on the wandering subspace problem ([20]). As an application, we establish a decomposition theorem for completely hyperexpansive operators.

Recall that $S$ in $\mathcal{B}(\mathcal{H})$ is analytic if $\bigcap_{n \geq 0} S^{n} \mathcal{H}=\{0\}$. To establish the promised Wold-type decomposition theorem, we need a couple of lemmas; one of these is borrowed from [20].

Lemma 4.1 ([20, Proposition 2.7]). If $S$ in $\mathcal{B}(\mathcal{H})$ is a left-invertible operator and if $S^{\prime}$ is the operator Cauchy dual to $S$ then the following duality formulae hold:

$$
\left(\bigcap_{n \geq 0} S^{\prime n} \mathcal{H}\right)^{\perp}=\bigvee_{n \geq 0} S^{n}\left(\operatorname{null}\left(S^{*}\right)\right), \quad\left(\bigcap_{n \geq 0} S^{n} \mathcal{H}\right)^{\perp}=\bigvee_{n \geq 0} S^{\prime n}\left(\operatorname{null}\left(S^{*}\right)\right) .
$$

Lemma 4.2. Let $S \in \mathcal{B}(\mathcal{H})$ be close to isometry. Set $\mathcal{H}_{\mathrm{u}}=\bigcap_{n \geq 0} S^{n} \mathcal{H}$. Then $S \mathcal{H}_{\mathrm{u}}=\mathcal{H}_{\mathrm{u}}, S^{*} \mathcal{H}_{\mathrm{u}}=\mathcal{H}_{\mathrm{u}}, S$ reduces $\mathcal{H}_{\mathrm{u}}$ and $\left.S\right|_{\mathcal{H}_{\mathrm{u}}}$ is unitary.

Proof. Let $\mathcal{U}$ be an AIR class. Let $S \in \mathcal{U} \cap \mathcal{B}(\mathcal{H})$ and set $\mathcal{H}_{\mathrm{u}}=\bigcap_{n \geq 0} S^{n} \mathcal{H}$. Clearly, $\mathcal{H}_{\mathrm{u}}$ is invariant for $S$. Indeed, $S \mathcal{H}_{\mathrm{u}}=\mathcal{H}_{\mathrm{u}}$. Thus $\left.S\right|_{\mathcal{H}_{\mathrm{u}}}$ is an invertible member of $\mathcal{U}$. Hence by Property I, $\left.S\right|_{\mathcal{H}_{\mathrm{u}}}$ is unitary. By Property II, $S$ reduces $\mathcal{H}_{\mathrm{u}}$. It is now obvious that $S^{*} \mathcal{H}_{\mathrm{u}}=\mathcal{H}_{\mathrm{u}}$. 
Theorem 4.3. Let $\mathcal{U}, \mathcal{V}$ be AIR classes, and let $\mathcal{C}: \mathcal{U} \rightarrow \mathcal{V}$ be the Cauchy map. If $T$ belongs to $\mathcal{U} \cap \mathcal{B}(\mathcal{H})$, then

$$
T=U \oplus A \quad \text { on } \mathcal{H}=\mathcal{H}_{\mathrm{u}} \oplus \mathcal{H}_{\mathrm{a}},
$$

where $U$ is unitary on $\mathcal{H}_{\mathrm{u}}, A$ is analytic on $\mathcal{H}_{\mathrm{a}}$, and $\mathcal{H}_{\mathrm{a}}=\bigvee_{n \geq 0} T^{n}\left(\operatorname{null}\left(T^{*}\right)\right)$.

Proof. Set $\mathcal{H}_{\mathrm{u}}=\bigcap_{n \geq 0} T^{n} \mathcal{H}$. By the preceding lemma,

$$
T=U \oplus A \quad \text { on } \mathcal{H}=\mathcal{H}_{\mathrm{u}} \oplus \mathcal{H}_{\mathrm{a}},
$$

where $U$ is unitary on $\mathcal{H}_{\mathrm{u}}$ and $A$ is analytic on $\mathcal{H}_{\mathrm{a}}$. To conclude the proof, it suffices to check that $\mathcal{H}_{\mathrm{a}}=\bigvee_{n \geq 0} T^{n}\left(\operatorname{null}\left(T^{*}\right)\right)$. Since $\mathcal{C}$ is a Cauchy map, the Cauchy dual operator $T^{\prime}$ belongs to $\mathcal{V}$. Since $T^{\prime}$ extends $A^{\prime}, A^{\prime}$ belongs to $\mathcal{V}$. By the previous lemma, $A^{\prime} \mathcal{H}_{\mathrm{u}}^{\prime}=\mathcal{H}_{\mathrm{u}}^{\prime}$ and $A^{\prime *} \mathcal{H}_{\mathrm{u}}^{\prime}=\mathcal{H}_{\mathrm{u}}^{\prime}$, where $\mathcal{H}_{\mathrm{u}}^{\prime} \equiv$ $\bigcap_{n \geq 0} A^{\prime n} \mathcal{H}_{\mathrm{a}}$. It is now easy to see that $A \mathcal{H}_{\mathrm{u}}^{\prime}=\mathcal{H}_{\mathrm{u}}^{\prime}$. Thus

$$
\mathcal{H}_{\mathrm{u}}^{\prime}=\bigcap_{n \geq 0} A^{n} \mathcal{H}_{\mathrm{u}}^{\prime} \subset \bigcap_{n \geq 0} A^{n} \mathcal{H}_{\mathrm{a}}=\{0\} .
$$

Consequently, $\mathcal{H}_{\mathrm{u}}^{\prime}=\{0\}$. By Lemma 4.1, $\mathcal{H}_{\mathrm{a}}=\bigvee_{n>0} A^{n}\left(\operatorname{null}\left(A^{*}\right)\right)$. Since $T$ extends $A$ and $\operatorname{null}\left(T^{*}\right)=\operatorname{null}\left(A^{*}\right), \mathcal{H}_{\mathrm{a}}=\bigvee_{n \geq 0} T^{n}\left(\operatorname{null}\left(T^{*}\right)\right)$ as desired.

Corollary 4.4. Let $\mathcal{U}, \mathcal{V}$ be AIR classes, and let $\mathcal{C}: \mathcal{U} \rightarrow \mathcal{V}$ be the Cauchy map. If $T$ in $\mathcal{U}$ is analytic, then for every invariant subspace $\mathcal{M}$ of $T$, one has $\mathcal{M}=\bigvee_{n \geq 0} T^{n}\left(\mathcal{M} \cap(T \mathcal{M})^{\perp}\right)$.

REMARK 4.5. We consider here two important cases of Corollary 4.4. In the case where $\mathcal{U}=\mathcal{U}_{1}, \mathcal{V}=\mathcal{U}_{2}$, and $\mathcal{C}=\mathcal{C}_{1,2}$ (see Example 3.10), the previous corollary recovers Beurling's theorem for Bergman-type operators ([4], [20]). In the case where $\mathcal{U}=\mathcal{U}_{2}, \mathcal{V}=\mathcal{U}_{3}$, and $\mathcal{C}=\mathcal{C}_{2,3}$ (Example 3.10), the corollary recovers Beurling's theorem for 2-hyperexpansive operators ([19], [6]).

Recall that a subspace $\mathcal{M}$ of $\mathcal{H}$ is said to be hyperinvariant for $T$ in $\mathcal{B}(\mathcal{H})$ if it is invariant for every operator $S$ in $\mathcal{B}(\mathcal{H})$ that commutes with $T$. The following corollary shows in particular that the class of power bounded complete hyperexpansions arises naturally out of the class of complete hyperexpansions.

Corollary 4.6. Let $T$ in $\mathcal{B}(\mathcal{H})$ be a completely hyperexpansive operator. Then there exist subspaces $\mathcal{M}, \mathcal{M}_{1}, \mathcal{M}_{2}$ of $\mathcal{H}$ such that:

(a) $\mathcal{M}$ is hyperinvariant for $T$,

(b) $\left.T\right|_{\mathcal{M}}=U \oplus S$ on $\mathcal{M}=\mathcal{M}_{1} \oplus \mathcal{M}_{2}$, where $U$ on $\mathcal{M}_{1}$ is unitary, $S$ on $\mathcal{M}_{2}$ is similar to a unilateral shift, and

$$
\mathcal{M}_{2}=\bigvee_{n \geq 0} T^{n}\left(\mathcal{M}_{2} \cap\left(T \mathcal{M}_{2}\right)^{\perp}\right),
$$

(c) $\left\|T^{n} h\right\| \rightarrow \infty$ as $n \rightarrow \infty$ for every non-zero $h$ in $\mathcal{M}^{\perp}$. 
In particular, a completely non-unitary power bounded 2-hyperexpansive $T$ is similar to the unilateral shift of multiplicity nullity $\left(T^{*}\right)$.

Proof. By Corollary 4.3 of [15], there exist a unique maximal tight PO measure $G$ on the interval $[0,1) \subset \mathbb{R}$ and a unique $B \in \mathcal{B}(\mathcal{H})$ such that

$$
T^{* n} T^{n}=I+n B+\int\left(1-x^{n}\right) d G(x) \quad(n \geq 1) .
$$

Set $\mathcal{M} \equiv \operatorname{null}(B)$ and write $\mathcal{H}=\mathcal{M} \oplus \mathcal{M}^{\perp}$. Let $S \in \mathcal{B}(\mathcal{H})$ with $S T=T S$ and let $h \in \mathcal{H}$ be such that $B h=0$. We first check that $B S h=0$.

By (4.4) and using $S T=T S, B h=0$, one has

$$
\begin{aligned}
\left\|T^{n} S h\right\|^{2} & =\left\langle T^{* n} T^{n} S h, S h\right\rangle \\
& =\left\langle\left(I+n B+\int_{[0,1)}\left(1-x^{n}\right) d G(x)\right) S h, S h\right\rangle \\
& =\|S h\|^{2}+n\left\|B^{1 / 2} S h\right\|^{2}+\left\langle\left(\int\left(1-x^{n}\right) d G(x)\right) S h, S h\right\rangle
\end{aligned}
$$

and

$$
\begin{aligned}
\left\|T^{n} S h\right\|^{2} & =\left\|S T^{n} h\right\|^{2} \\
& \leq\|S\|^{2}\left\|T^{n} h\right\|^{2}=\|S\|^{2}\left\langle T^{* n} T^{n} h, h\right\rangle \\
& =\|S\|^{2}\left\langle\left(I+n B+\int_{[0,1)}\left(1-x^{n}\right) d G(x)\right) h, h\right\rangle \\
& =\|S\|^{2}\left\{\|h\|^{2}+\left\langle\left(\int_{[0,1)}\left(1-x^{n}\right) d G(x)\right) h, h\right\rangle\right\} .
\end{aligned}
$$

Therefore

$$
\begin{aligned}
\|S h\|^{2}+n\left\|B^{1 / 2} S h\right\|^{2}+ & \left\langle\left(\int_{[0,1)}\left(1-x^{n}\right) d G(x)\right) S h, S h\right\rangle \\
& \leq\|S\|^{2}\left\{\|h\|^{2}+\left\langle\left(\int_{[0,1)}\left(1-x^{n}\right) d G(x)\right) h, h\right\rangle\right\} .
\end{aligned}
$$

An easy application of the Lebesgue monotone convergence theorem yields

$$
\begin{aligned}
& \|S h\|^{2}+\lim _{n \rightarrow \infty} n\left\|B^{1 / 2} S h\right\|^{2}+\langle G([0,1) S h, S h\rangle \\
& \leq\|S\|^{2}\left(\|h\|^{2}+\langle G([0,1)) h, h\rangle\right) .
\end{aligned}
$$

Consequently, $\left\|B^{1 / 2} S h\right\|=0$ and hence $B S h=0$. This proves (a).

Using the representation (4.4), it can be easily seen that $\left.T\right|_{\mathcal{M}}$ is power bounded. Hence $\left.T\right|_{\mathcal{M}}$ is a power bounded complete hyperexpansion. Let $h$ be a non-zero vector in $\mathcal{M}^{\perp}$. Then $B h \neq 0$ and hence using (4.4), one has $\left\|T^{n} h\right\| \rightarrow \infty$ as $n \rightarrow \infty$. To conclude the proof, it suffices to show that $\left.T\right|_{\mathcal{M}}$ can be decomposed as a direct sum of a unitary and an operator similar to a 
unilateral shift. Indeed, we show that any power bounded 2-hyperexpansion can be decomposed in this manner. To see that, let $T$ in $\mathcal{B}(\mathcal{H})$ be a power bounded expansion. By the standard theory (see for example, Section 3.2 of [17]), a power bounded expansion is similar to an isometry. Since we need to know the intertwining operator, we include the elementary details. Notice that $\left\{T^{* n} T^{n}\right\}_{n \geq 0}$ is a non-decreasing, bounded sequence of positive expansions, so it converges strongly in $\mathcal{B}(\mathcal{H})([17])$. Hence there exists a positive operator $A$ in $\mathcal{B}(\mathcal{H})$ such that

$$
T^{* n} T^{n} \rightarrow A(\mathrm{SOT}) \quad \text { as } n \rightarrow \infty .
$$

Since $T^{* n} T^{n}$ is a positive expansion for every $n \geq 0$, so is $A$. By uniqueness of strong limits, $\left\{T^{* n} T^{* k} T^{k} T^{n}\right\}_{n, k \geq 0}$ and $\left\{T^{* k} T^{k}\right\}_{k \geq 0}$ converge to $A$ for any $n \geq 0$. Therefore $T^{* n} A T^{n}=A$ for every $n \geq 0$. Consequently,

$$
\left\|A^{1 / 2} T^{n} h\right\|^{2}=\left\langle T^{* n} A T^{n} h, h\right\rangle=\langle A h, h\rangle=\left\|A^{1 / 2} h\right\|^{2}
$$

for every $h \in \mathcal{H}$ and $n \geq 0$. Define an isometry $V$ in $\mathcal{B}(\mathcal{H})$ as follows. For an arbitrary $y \in \mathcal{H}$, let $x \in \mathcal{H}$ be such that $y=A x$ and define $V y=A^{1 / 2} T A^{1 / 2} x$. It is easy to check that $T=A^{-1 / 2} V A^{1 / 2}$ and that $V$ is isometric.

Now suppose that $T$ in $\mathcal{B}(\mathcal{H})$ is a power bounded 2-hyperexpansion. By Example 3.10 and Theorem $4.3, \mathcal{H}_{\mathrm{u}}$ is a reducing subspace for $T, U=\left.T\right|_{\mathcal{H}_{\mathrm{u}}}$ is unitary, and $\mathcal{H} \ominus \mathcal{H}_{\mathrm{u}}=\bigvee_{n \geq 0} T^{n}\left(\mathcal{H} \cap(T \mathcal{H})^{\perp}\right)$. Thus it suffices to check that $C=\left.T\right|_{\mathcal{H}_{\mathrm{u}}^{\perp}}$ is similar to a unilateral shift. By the discussion in the preceding paragraph, there exist an isometry $V$ and a positive expansion $B$ in $\mathcal{B}(\mathcal{H})$ such that

$$
B T=V B, \quad B^{2}=\lim _{n \rightarrow \infty} T^{* n} T^{n}(\mathrm{SOT}) .
$$

Since $T$ reduces $\mathcal{H}_{\mathrm{u}}$, we must have $T^{* n} T^{n} \mathcal{H}_{\mathrm{u}} \subset \mathcal{H}_{\mathrm{u}}$ for every $n \geq 0$. Letting $n \rightarrow \infty$ leads to $B^{2} \mathcal{H}_{\mathrm{u}} \subset \mathcal{H}_{\mathrm{u}}$. The spectral theorem yields $B \mathcal{H}_{\mathrm{u}} \subset \mathcal{H}_{\mathrm{u}}$. Because $B$ is a positive expansion, $B$ reduces $\mathcal{H}_{\mathrm{u}}$, and $\left.B\right|_{\mathcal{H}_{\mathrm{u}}},\left.B\right|_{\mathcal{H}_{\mathrm{u}}^{\perp}}$ are positive expansions. Set $\mathcal{H}_{\infty} \equiv \bigcap_{n \geq 0} V^{n} \mathcal{H}$. We claim that $B \mathcal{H}_{\mathrm{u}} \subset \mathcal{H}_{\infty}$ and $B \mathcal{H}_{\mathrm{u}}^{\perp} \subset \mathcal{H}_{\infty}^{\perp}$. To see the first inclusion, let $x \in \mathcal{H}_{\mathrm{u}}$. Then for every $n \geq 0$, there exists $x_{n} \in \mathcal{H}$ such that $x=T^{n} x_{n}$. Thus

$$
B x=B T^{n} x_{n}=B T^{n} B^{-1}\left(B x_{n}\right)=V^{n} B x_{n} \in V^{n} \mathcal{H}
$$

for every $n \geq 0$. Hence $B \mathcal{H}_{\mathrm{u}} \subset \mathcal{H}_{\infty}$. Next let $x \in \mathcal{H}_{\mathrm{u}}^{\perp}$ and $y \in \mathcal{H}_{\infty}$. Since for every $n \geq 0, y=V^{n} x_{n}$ for some $x_{n} \in \mathcal{H}$, one has

$$
\langle B x, y\rangle=\langle x, B y\rangle=\left\langle x, B^{2}\left(B^{-1} V^{n} B\right) B^{-1} x_{n}\right\rangle=\left\langle x, B^{2} T^{n} B^{-1} x_{n}\right\rangle
$$

for every $n \geq 0$. Since $T^{n} B^{-1} x_{n} \in \mathcal{H}_{\mathrm{u}}$ and $B \mathcal{H}_{\mathrm{u}} \subset \mathcal{H}_{\mathrm{u}}$, we must have $B^{2}\left(T^{n} B^{-1} x_{n}\right)$ in $\mathcal{H}_{\mathrm{u}}$. Hence $\langle B x, y\rangle=0$ for every $y \in \mathcal{H}_{\infty}$. Consequently, $B \mathcal{H}_{\mathrm{u}}^{\perp} \subset \mathcal{H}_{\infty}^{\perp}$. This completes the proof of the claim. 
By the von Neumann-Wold-Kolmogorov decomposition theorem for isometries, one has

$$
V=V_{1} \oplus V_{2} \quad \text { on } \mathcal{H}=\mathcal{H}_{\infty} \oplus \mathcal{H}_{\infty}^{\perp},
$$

where $V_{1}$ is unitary and $V_{2}$ is the unilateral shift of multiplicity nullity $\left(V^{*}\right)$ ([10]). Now it follows readily from $B T=V B$ and the inclusions $B \mathcal{H}_{\mathrm{u}} \subset \mathcal{H}_{\infty}$, $B \mathcal{H}_{\mathrm{u}}^{\perp} \subset \mathcal{H}_{\infty}^{\perp}$ established in the preceding paragraph that

$$
\left.T\right|_{\mathcal{H}_{\mathrm{u}}^{\perp}}=\left(\left.B\right|_{\mathcal{H}_{\mathrm{u}}^{\perp}}\right)^{-1} V_{2}\left(\left.B\right|_{\mathcal{H}_{\mathrm{u}}^{\perp}}\right)
$$

that is, $\left.T\right|_{\mathcal{H}_{\mathrm{u}}^{\perp}}$ is similar to $V_{2}$ as desired.

REMARK 4.7. Let $T$ and $\mathcal{M}$ be as in the statement of Corollary 4.6. We consider here two extreme cases:

(a) The case in which $\mathcal{M}=\mathcal{H}$ and $T$ is a power bounded complete hyperexpansion. In this case, there exist a unitary $U$, an invertible expansion $B$, a unilateral shift $V$ and a reducing subspace $\mathcal{L}$ for $T$ such that

$$
T=U \oplus B^{-1} V B \quad \text { on } \mathcal{H}=\mathcal{L} \oplus \mathcal{L}^{\perp},
$$

where $B$ is the restriction of the positive square root of the SOT limit $\lim _{n \rightarrow \infty} T^{* n} T^{n}$ to $\mathcal{L}^{\perp}$. By Theorem 5.3 of [15], there exists a maximal tight $\mathrm{PO}$ measure $G$ on $[0,1) \subset \mathbb{R}$ such that $T^{* n} T^{n}=$ $I+\int_{[0,1)}\left(1-x^{n}\right) d G(x)$. By a routine application of the Lebesgue monotone convergence theorem, $T^{* n} T^{n} \rightarrow I+G([0,1))$ (SOT) as $n \rightarrow \infty$. Hence $B=\left.(I+G([0,1)))^{1 / 2}\right|_{\mathcal{L}^{\perp}}$.

(b) The case in which $\mathcal{M}=\{0\}$ and $\left\|T^{n} h\right\| \rightarrow \infty$ for every non-zero $h$ in $\mathcal{H}$. One may refer to such operators as strict complete hyperexpansions. The Dirichlet shift $T:\{\sqrt{(n+2) /(n+1)}\}_{n \geq 0}$ given by $T e_{n}=\sqrt{(n+2) /(n+1)} e_{n+1}$ is an example of a strict complete hyperexpansion, where $\left\{e_{n}\right\}_{n \geq 0}$ is an orthonormal basis of $\mathcal{H}$. Using the representation (4.4), it is easy see that $\|T h\|>\|h\|$ for every non-zero vector $h$ in $\mathcal{H}$. Note also that a strict complete hyperexpansion is completely non-isometric (that is, has no non-trivial isometric direct summand), and hence completely non-unitary. The Dirichlet shift may play a significant role in a model theory for strict complete hyperexpansions.

We conclude this section with an application of Corollary 4.6. Let $T$ be a completely non-unitary power bounded 2-hyperexpansion. For $S$ in $\mathcal{B}(\mathcal{H})$, let $P^{\infty}(S)$ denote the weak* closed algebra generated by $S$ and the identity operator $I$. Let nullity $\left(T^{*}\right)=n \in \mathbb{N} \cup\{\infty\}$ and let $\mathcal{S}$ denote the unilateral shift. Recall that a unilateral shift of multiplicity $n$ is unitarily equivalent to the direct sum $\mathcal{S}^{(n)}$ of $n$ copies of $\mathcal{S}([10])$. By Theorem 6.2 of Chapter I of [10], the map $\phi \mapsto \phi(\mathcal{S})^{(n)}$ is an isometric algebraic isomorphism of $\mathcal{H}^{\infty}(\mathbb{D})$ 
onto $P^{\infty}\left(\mathcal{S}^{(n)}\right)$ that is a weak* homeomorphism. In view of Remark 4.9(a), one may define a functional calculus for $T$ by $\phi(T)=B^{-1} \phi(\mathcal{S})^{(n)} B$ for any $\phi$ in the Hardy space $\mathcal{H}^{\infty}\left(\mathbb{D}_{1}\right)$. The mapping $\psi_{T}: \mathcal{H}^{\infty}\left(\mathbb{D}_{1}\right) \rightarrow P^{\infty}(T)$ given by $\psi_{T}(\phi)=\phi(T)$ is evidently a bounded algebraic homomorphism and a weak ${ }^{*}$ homeomorphism onto $P^{\infty}(T)$. Moreover, $\psi_{T}$ is injective. To see that, let $h \in \mathcal{H}$ with $\|h\|=1$ and let $\phi \in \mathcal{H}^{\infty}\left(\mathbb{D}_{1}\right)$. Using $\|B h\| \geq 1$, one can easily check that

$$
\left\|\psi_{T}(\phi) h\right\| \geq\|B\|^{-1}\left\|\phi(\mathcal{S})^{(n)} \frac{B h}{\|B h\|}\right\| .
$$

Since $B$ is onto and $\left\|\phi(\mathcal{S})^{(n)}\right\|=\|\phi\|_{\infty}$, the preceding inequality yields

$$
\begin{aligned}
\left\|\psi_{T}(\phi)\right\| & =\sup _{h \in \mathcal{H},\|h\|=1}\left\|\psi_{T}(\phi) h\right\| \\
& \geq\|B\|^{-1} \sup _{h \in \mathcal{H},\|h\|=1}\left\|\phi(\mathcal{S})^{(n)} \frac{B h}{\|B h\|}\right\| \\
& =\|B\|^{-1}\left\|\phi(\mathcal{S})^{(n)}\right\|=\|B\|^{-1}\|\phi\|_{\infty} .
\end{aligned}
$$

In particular, $\psi_{T}$ is injective. An immediate consequence of this functional calculus is that $\sigma(\phi(T))=\overline{\phi\left(\mathbb{D}_{1}\right)}$ for any $\phi \in \mathcal{H}^{\infty}\left(\mathbb{D}_{1}\right)$ (refer to Sections 4,6 of Chapter I of [10]). Summing up:

COROLlary 4.8. Let $T$ be a completely non-unitary power bounded 2hyperexpansion, and let $\psi_{T}: \mathcal{H}^{\infty}\left(\mathbb{D}_{1}\right) \rightarrow P^{\infty}(T)$ be as in the preceding paragraph. Then $\psi_{T}$ is an injective bounded algebraic homomorphism and a weak ${ }^{*}$ homeomorphism onto $P^{\infty}(T)$. In particular, $\sigma(\phi(T))=\overline{\phi\left(\mathbb{D}_{1}\right)}$ for any $\phi \in \mathcal{H}^{\infty}\left(\mathbb{D}_{1}\right)$.

5. Concluding remarks. In this section we briefly discuss one possible generalization of some results established in Section 3. For that purpose, we will have to introduce some more restriction classes, which satisfy properties slightly weaker than Properties I and II (see Definition 2.1). To be more precise, we consider those restriction classes $\mathcal{N}$ for which the following hold.

Property I*. If $S$ belongs to $\mathcal{N}$, and $S$ is invertible, then $S$ is normal.

Property II*. If $S$ belongs to $\mathcal{N}$, and $\mathcal{M}$ is a non-zero invariant subspace of $S$ such that $\left.S\right|_{\mathcal{M}}$ is normal, then $S$ reduces $\mathcal{M}$.

Note that every AIR class has Properties I*, II*. Thus the restriction classes discussed in Example 2.3 have these properties. Moreover, we have 
EXAMPLE 5.1. Set

$\mathcal{N}_{1} \equiv\left\{T: T\right.$ is hyponormal with $\sigma_{\text {ap }}(T) \subset \partial \mathbb{D}_{r}$ for some $\left.r>0\right\}$,

$\mathcal{N}_{2} \equiv\{T: T$ is alternatingly hyperexpansive with

$$
\left.r^{-1} T \text { expansive and } \sigma_{\mathrm{ap}}(T) \subset \partial \mathbb{D}_{r} \text { for some } r>0\right\} .
$$

Then $\mathcal{N}_{1}$ and $\mathcal{N}_{2}$ are restriction classes and have Properties I*, II*.

Verification. Since the argument is similar to one in Example 2.3(1), we omit the verification that $\mathcal{N}_{1}, \mathcal{N}_{2}$ have Property I*. To see that they have Property $\mathrm{II}^{*}$, let $T$ in $\mathcal{B}(\mathcal{H})$ be such that $r^{-1} T$ is either contractive or expansive, with $\sigma_{\text {ap }}(T) \subset \partial \mathbb{D}_{r}$. Suppose further that $\left.T\right|_{\mathcal{M}}$ is normal for some non-zero invariant subspace $\mathcal{M}$ of $T$. Using the spectral mapping property of bounded linear operators $([9])$, it is easy to see that $\left.r^{-1} T\right|_{\mathcal{M}}$ is unitary. Since $r^{-1} T$ is either contractive or expansive, by Lemma 2.5, $T$ reduces $\mathcal{M}$. It is now clear that $\mathcal{N}_{2}$ has Property II*. Since for any hyponormal $S$, the norm of $S$ is equal to its spectral radius, an operator $T$ in $\mathcal{N}_{1}$ satisfies $\left\|r^{-1} T\right\| \leq 1$. Hence by the preceding discussion, $\mathcal{N}_{1}$ also has Property II*. This completes the verification of Example 5.1.

The following theorem generalizes Theorem 3.2 of Section 3 .

Theorem 5.2. Let $\mathcal{N}$ be a restriction class with Properties $\mathrm{I}^{*}, \mathrm{II}^{*}$. Let $r>0$. If $T$ belongs to $\mathcal{N} \cap \mathcal{B}(\mathcal{H})$, then

$$
T=N \oplus C \quad \text { on } \mathcal{H}=\mathcal{H}_{\mathrm{n}} \oplus \mathcal{H}_{\mathrm{c}},
$$

where $N$ is normal on $\mathcal{H}_{\mathrm{n}}, C$ is completely non-normal on $\mathcal{H}_{\mathrm{c}}$, and $\mathcal{H}_{\mathrm{c}}$ is equal to $\bigvee\left\{\operatorname{null}\left(C^{*}-\mu I\right): \mu \in \mathbb{D}_{r}\right\}$. If in addition $T$ is completely non-normal then $T^{*}$ admits a dense set of cyclic vectors.

Proof. For the first part, imitate the proof of Theorem 3.2. The remaining part can be derived from Theorem 3 of [8].

Corollary 5.3. If $T$ in $\mathcal{B}(\mathcal{H})$ belongs to one of the restriction classes $\mathcal{N}_{1}, \mathcal{N}_{2}$ (see Example 5.1), and if $T$ is an m-multicyclic completely nonnormal operator, then $T^{*}$ belongs to $\mathcal{B}_{m}\left(\mathbb{D}_{r}\right)$ for some $r>0$.

Proof. The desired conclusion follows from Example 5.1, Lemma 2.6, and the preceding theorem.

There are some important and natural restriction classes to which the preceding results are not applicable.

EXAMPLE 5.4. The restriction classes

$$
\begin{aligned}
& \mathcal{R}_{1} \equiv\{T: T \text { is a } p \text {-isometry for some positive integer } p\}, \\
& \mathcal{R}_{2} \equiv\{T: T \text { is alternatingly hyperexpansive }\}
\end{aligned}
$$

do not have Property I*. 
Verification. We first check that $\mathcal{R}_{1}$ does not have Property I*. Consider the operator

$$
T \equiv \bigoplus_{n=1}^{\infty}\left(\begin{array}{cc}
\alpha_{n} & c \\
0 & \alpha_{n}
\end{array}\right) \quad \text { on } \mathcal{H} \equiv \bigoplus_{n=1}^{\infty} \mathbb{C}^{2},
$$

where $c>0$ and $\left\{\alpha_{n}\right\}_{n \geq 1}$ is a sequence in $\partial \mathbb{D}_{1}$. It is not difficult to see that $T$ is an invertible 3 -isometry which is not normal. Thus $\mathcal{R}_{1}$ does not have Property I*.

To construct an invertible alternating hyperexpansion which is not normal, we proceed as follows. Let $A(1 / 2,1)$ be the open annulus $\{z \in \mathbb{C}: 1 / 2<$ $|z|<1\}$. Let $L^{2}(A(1 / 2,1))$ denote the Lebesgue space of measurable functions square-integrable with respect to the area measure restricted to $A(1 / 2,1)$. Let $L_{\mathrm{a}}^{2}(A(1 / 2,1))$ be the Bergman space

$$
\left\{f \in L^{2}(A(1 / 2,1)): f \text { is analytic on } A(1 / 2,1)\right\} .
$$

Then $L_{\mathrm{a}}^{2}(A(1 / 2,1))$ is a Hilbert space. Let $B$ denote the operator of multiplication by the coordinate function $z$ on $L_{\mathrm{a}}^{2}(A(1 / 2,1))$. Then $B$ is an invertible subnormal contraction which is not normal (see Section 8 of Chapter II of [10]). Since the inverse of an invertible subnormal contraction is alternatingly hyperexpansive ([21, Proposition 4.1(i)]), it follows that $B^{-1}$ is an invertible alternating hyperexpansion which is not normal. This completes the verification of Example 5.4.

REMARK 5.5. Since the operator $B$ in the preceding example is an invertible hyponormal operator which is not normal, Property $\mathrm{I}^{*}$ is violated for the restriction class $\{T: T$ is hyponormal $\}$.

At this point, we mention that as in the case of the AIR classes $\mathcal{U}_{i}(i=$ $1,2)$ the Wold-type decomposition theorems for the restriction classes $\mathcal{U}_{4}, \mathcal{N}_{1}$ demand the existence of the corresponding Cauchy maps. This is one of the reasons one should know more about the operators Cauchy dual to $\mathrm{m}$ isometries $(m \geq 3)$ and alternating hyperexpansions ([20], [6]).

Acknowledgments. The author expresses his gratitude for the hospitality of the Indian Institute of Technology, Powai, where the substantial part of this work was done. The author would specially like to thank Prof. B. V. Limaye and Prof. Ameer Athavale for their constant encouragement.

\section{References}

[1] J. Agler, A disconjugacy theorem for Toeplitz operators, Amer. J. Math. 112 (1990), $1-14$. 
[2] J. Agler and M. Stankus, m-isometric transformations of Hilbert spaces, I, II, III, Integral Equations Operator Theory 21, 23, 24 (1995, 1995, 1996), 383-429, 1-48, 379-421.

[3] A. Aleman, The multiplication operators on Hilbert spaces of analytic functions, Habilitationsschrift, Fernuniversität Hagen, 1993.

[4] A. Aleman, S. Richter and C. Sundberg, Beurling's theorem for the Bergman space, Acta Math. 177 (1996), 275-310.

[5] A. Athavale, On completely hyperexpansive operators, Proc. Amer. Math. Soc. 124 (1996), 3745-3752.

[6] S. Chavan, On operators Cauchy dual to 2-hyperexpansive operators, Proc. Edinburgh Math. Soc. 50 (2007), 637-652.

[7] K. Clancey, Completeness of eigenfunctions of seminormal operators, Acta Sci. Math. (Szeged) 39 (1977), 31-37.

[8] K. Clancey and D. Rogers, Cyclic vectors and seminormal operators, Indiana Univ. Math. J. 27 (1978), 689-696.

[9] J. Conway, A Course in Functional Analysis, Springer, New York, 1997.

[10] —, The Theory of Subnormal Operators, Math. Surveys Monogr. 36, Amer. Math. Soc., Providence, RI, 1991.

[11] M. Cowen and R. Douglas, Complex geometry and operator theory, Acta Math. 141 (1978), 187-261.

[12] N. Feldman, Pure subnormal operators have cyclic adjoints, J. Funct. Anal. 162 (1999), 379-399.

[13] R. Gethner and J. Shapiro, Universal vectors for operators on spaces of holomorphic functions, Proc. Amer. Math. Soc. 100 (1987), 281-288.

[14] D. Herrero, On multicyclic operators, Integral Equations Operator Theory 1 (1978), 57-102.

[15] Z. Jabłoński, Complete hyperexpansivity, subnormality and inverted boundedness conditions, ibid. 44 (2002), 316-336.

[16] Z. Jabłoński and J. Stochel, Unbounded 2-hyperexpansive operators, Proc. Edinburgh Math. Soc. 44 (2001), 613-629.

[17] C. Kubrusly, An Introduction to Models and Decompositions in Operator Theory, Birkhäuser, Boston, MA, 1997.

[18] B. Morrel, A decomposition for some operators, Indiana Univ. Math. J. 23(1973), 497-511.

[19] S. Richter, Invariant subspaces of the Dirichlet shift, J. Reine Angew. Math. 386 (1988), 205-220.

[20] S. Shimorin, Wold-type decompositions and wandering subspaces for operators close to isometries, ibid. 531 (2001), 147-189.

[21] V. Sholapurkar and A. Athavale, Completely and alternatingly hyperexpansive operators, J. Operator Theory 43 (2000), 43-68.

[22] D. Yakubovich, Dual piecewise analytic bundle shift models of linear operators, J. Funct. Anal. 136 (1996), 294-330.

Indian Institute

of Science, Education \& Research, Pune

Pune 411008, India

E-mail: sl.chavan@iiserpune.ac.in 